Diabetologe 2011 · 7:8-8

DOI 10.1007/s11428-011-0683-2

(c) Springer-Verlag 2011

\section{Halle}

Innere Medizin - Kardiologie - Sportmedizin, Präventive und Rehabilitative

Sportmedizin, Klinikum rechts der Isar, Technische Universität München

\title{
Sport und Diabetes
}

Kürzlich haben das American Collage of Sports Medicine und die American Diabetes Association in einem gemeinsamen Positionspapier die Bedeutung körperlicher Aktivität in der Prävention und Therapie des Diabetes mellitus bekräftigt (MSSE 2010). Die wissenschaftliche Bedeutung dieses Themas wird auch daran erkennbar, dass ungefähr ein Viertel aller Publikationen zum Thema „Diabetes“ auch den Stellenwert „körperliche Aktivität" untersucht bzw. mit diskutiert hat. Doch gibt es deutliche Unterschiede zwischen dem Typ-1- und dem Typ2-Diabetes-mellitus. So ist die Datenlage gerade auch im Hinblick auf körperliche Aktivität und Langzeitprognose beim Typ-1-Diabetes nach wie vor sehr dürftig, während zum Typ-2-Diabetes drei- bis viermal so viele Studien publiziert werden.

Sicherlich ist dieses der vergleichsweise deutlich höheren und nach wie vor steigenden Prävalenz des Typ-2-Diabetes-mellitus geschuldet. Außerdem spielt auch eine wesentliche Rolle, dass eine unregelmäßige sportliche Betätigung beim Typ-1-Diabetes häufig zu Stoffwechselkapriolen führt, eher die Blutzuckereinstellung erschwert sowie Langzeit-Blutzuckerwerte verschlechtert. Im Gegensatz hierzu kann körperliche Aktivität beim Typ-2-Diabetes die pathophysiologisch erhöhte Insulinresistenz und damit die Stoffwechselsituation substanziell verbessern. Insgesamt ist somit das Training bei einem Typ-1-Diabetes-Patienten deutlich individueller zu gestalten und stellt eine größere Herausforde- rung an Arzt und Patient dar als das Training beim Typ-2-Diabetes.

\section{In der Praxis ist es schwierig, Diabetespatienten zu regelmäßiger körperlicher Aktivität zu motivieren}

Ein wesentliches Problem verbleibt: In der praktischen Versorgung ist es vielfach schwierig, Patienten mit Typ-2-Diabetes-mellitus zu motivieren, regelmäßig körperliche Aktivität aufzunehmen und eine Lebensstilumstellung langfristig fortzuführen. Entsprechendes zeigt sich in 2 großen randomisierten Studien mit 8000 Patienten zu Lebensstilumstellung (Ernährung und Bewegung) bei systolischer Herzinsuffizienz (HF ACTION) und Typ-2-Diabetes mellitus (Look-AHEAD-Studie). So kann der Umfang an körperlicher Aktivität im Sinne der empfohlenen 30 Minuten pro Tag nur für das erste Jahr ausreichend eingehalten werden und nimmt nachfolgend sukzessive wieder ab. Der Effekt des Trainings auf klinische Parameter wird dadurch für diese Patienten über die erfolgreiche Interventionsphase hinaus kaum beeinflusst. Patienten, die das Programm langfristig umsetzen können, profitieren substanziell auch hinsichtlich ihrer Prognose.

Ziel in der Therapie des Diabetespatienten ist die Konversion vom „passiven“ zum „aktiven“ Patienten. Um dieses zu erreichen, muss neben der Selbstdisziplin des Patienten ein strukturiertes Lebensstilprogramm initiiert werden. Die Anleitung zu körperlichem Training darf nicht allein darin bestehen, einen allgemeinen Hinweis wie „Treppensteigen statt Fahrstuhl“ zu geben, sondern muss dezidierte Empfehlungen zu Art, Ausdauer, Intensität und Umfang der körperlichen Aktivität beinhalten. Wichtig ist die gleichzeitige enge Kontrolle durch den Arzt und das betreuende Team. Für die Initiierung und Durchführung dieses Konzeptes sind sportmedizinische und psychologische Vorkenntnisse des Hausarztes, Diabetologen und Kardiologen notwendig. Aus diesem Grund ist es eine zentrale Aufgabe, diese Kenntnisse in Vorlesungen, Vorträgen und Publikationen zu vermitteln. Gleichzeitig müssen im deutschen Gesundheitssystem Strukturen geschaffen werden, die entsprechende Konzepte der erfolgreichen Lebensstilintervention fördern. Hierzu gehört auch ganz wesentlich eine entsprechende Honorierung für alle Beteiligten - ein noch langer Weg, der sich sicherlich für unsere $\mathrm{Pa}$ tienten lohnt zu gehen.

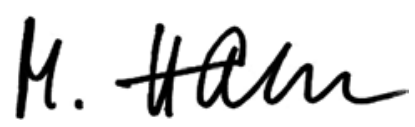

M. Halle

\section{Korrespondenzadresse \\ Prof. Dr. M. Halle}

Innere Medizin - Kardiologie - Sportmedizin, Präventive und Rehabilitative Sportmedizin, Klinikum rechts der Isar, Technische Universität München Connollystr. 32, 80809 München

halle@sport.med.tum.de 